\title{
Optimization of a large aperture dipole magnet for baryonic matter studies at Nuclotron
}

\author{
P.G. Akishin ${ }^{\dagger}$, A.Yu. Isupov ${ }^{\dagger}$, A.N. Khrenov ${ }^{\dagger}$, P.K. Kurilkin ${ }^{\dagger}$, \\ V.P. Ladygin ${ }^{\dagger, \neq}, 1$, S.M. Piyadin ${ }^{\dagger}$ and N.D. Topilin ${ }^{\dagger}$ \\ $\dagger$ Joint Institute for Nuclear Research, 141980 Dubna, Russia \\ $\ddagger$ E-mail:vladygin@jinr.ru
}

\begin{abstract}
The aperture of the dipole magnet SP41 has been enlarged for the studies of dense baryonic matter properties at Nuclotron. The homogeneity of the magnetic field in the magnet centre has been improved. The measurement results of the magnetic field components and integral are compared with results of 3D TOSCA calculations.
\end{abstract}

PACS: 07.55.Db, 29.30.Aj

\section{Introduction}

The major goal in the studies of the dense baryonic matter at Nuclotron (BM@N project) [1] is the measurement of strange and multi-strange baryons and mesons in heavy ion collisions at the beam energies between 2 and 6 A.GeV [2]. The physics program can be extended to the measurements of the in-medium effects for strange particles decaying in hadronic modes [3], hard probes and correlations [4], spin and polarization effects [5, 6] etc.

For these purposes an experimental set-up will be installed at the $6 \mathrm{~V}$ beamline in the fixed-target hall of the Nuclotron. The $6 \mathrm{~V}$ beamline contains the quadrupole lenses doublet, two dipole magnets allowing to correct the beam position in the vertical and horizontal planes, and large aperture SP41 dipole magnet for the momentum measurements [1]. The first results with the relativistic deuteron [7] and carbon [8] beams are demonstrated the feasibility of the dense baryonic matter studies with light nuclei using $6 \mathrm{~V}$ beamline infrastructure.

The modified SP41 dipole magnet will be used as an analyzing magnet [1]. Initially, the length of the magnet pole along the beam was $2.50 \mathrm{~m}$, width in the horizontal direction was $1.70 \mathrm{~m}$ and the height was about $0.75 \mathrm{~m}$. This

\footnotetext{
${ }^{1}$ Corresponding author
} 


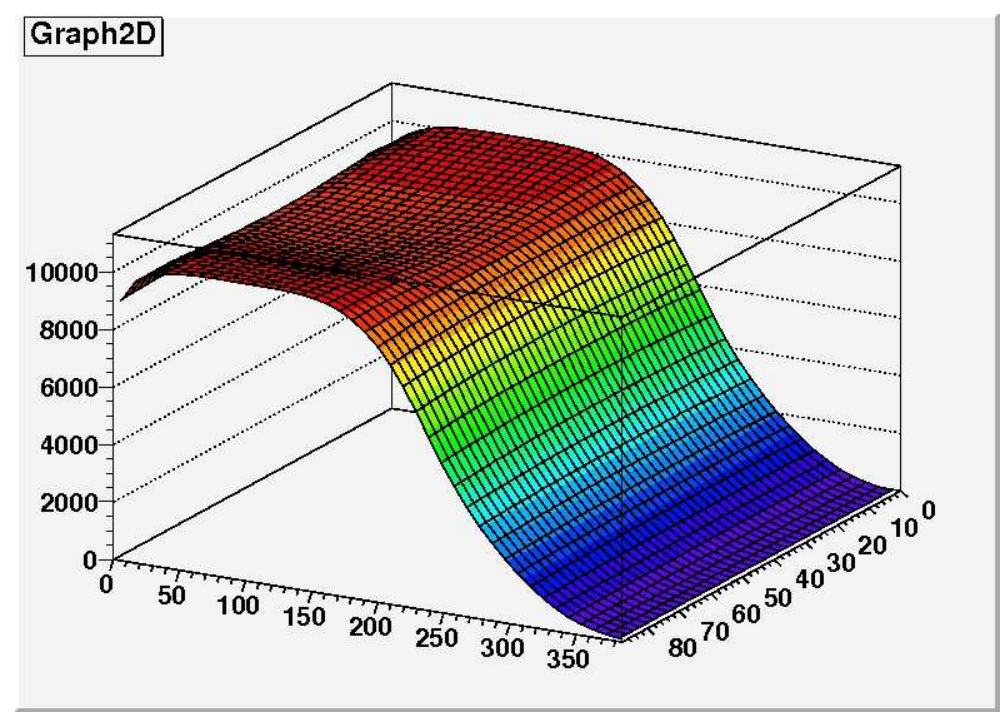

Figure 1: The vertical component $B_{y}$ of the magnetic field in the centre of the SP41 magnet before the modification.

magnet had also a hole for the photo-camera in the upper pole since it was used previously for the experiments with streamer chamber. As the result the magnetic field components are demonstrated the non-uniform behaviour. The $2 D$ dependence of the magnetic field vertical component $B_{y}$ of the nonmodified magnet SP41 in $X Z$ plane is shown in Fig.1.

However, the detection of multi-strange baryons requires the large aperture silicon tracking system placement into the homogeneous magnetic field [1, 2, 6]. The SP41 magnet was modified to satisfy this requirement, namely, the distance between the poles has been enlarged up to $1.05 \mathrm{~m}$ and the hole in the upper pole and horizontal beams has been filled by the steel-15. In this paper the results of the magnetic field measurements are presented for the modified dipole magnet SP41. These results are compared with the 3D magnetic field TOSCA calculations.

\section{Magnetic field calculations}

The magnetic field calculations for modified SP41 dipole magnet has been performed using 3D TOSCA code [9]. Steel-10, steel-15 and copper were taken as the material for the yoke, magnet poles and coils, respectively. The coordinate system was chosen as following: $X$ axis is perpendicular to the 


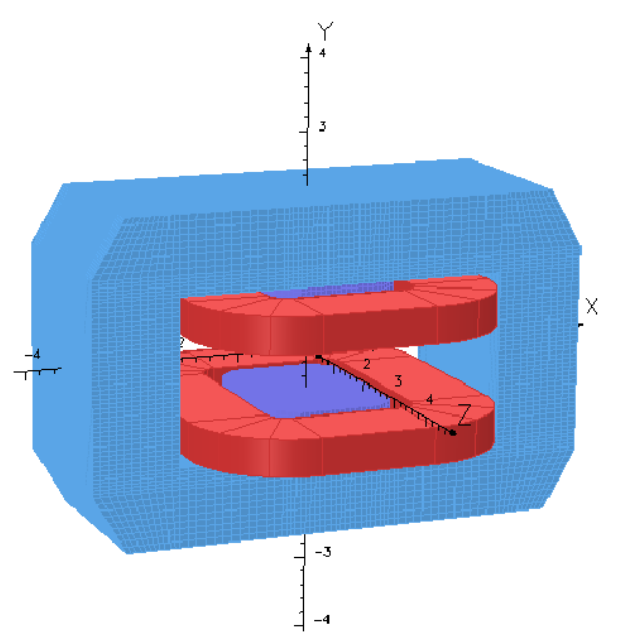

Figure 2: SP41 dipole magnet model for the magnetic field TOSCA [9] calculations.

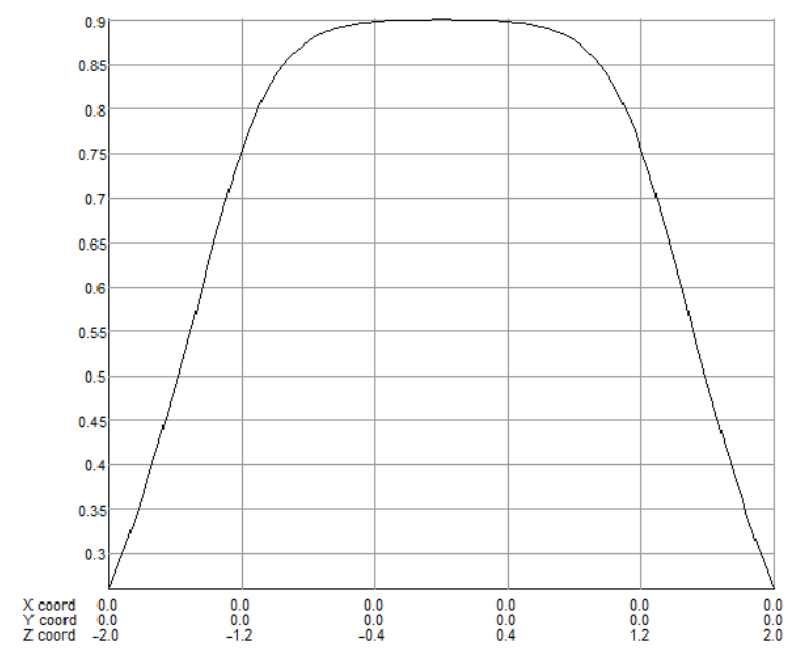

Figure 3: The 3D TOSCA calculation results for the magnetic field vertical component $B_{y}$ in the centre of the modified magnet SP41 along the beam direction. 
beam direction in the horizontal plane, $Y$ is vertical and $Z$ is along the beam and parallel to the magnet poles. The 3D model of the modified SP41 dipole magnet for the magnetic field TOSCA calculations is presented in Fig. 2.

The 3D TOSCA calculation results for the magnetic field vertical component $B_{y}$ in the centre of the modified magnet SP41 along the beam direction are demonstrated in Fig. 3. The current in the coils was taken as $1900 \mathrm{~A}$. The maximal value of the vertical component $B_{y}$ was found $\sim 0.9 \mathrm{~T}$. The field integral is $\sim 2.9 \mathrm{~T} \cdot \mathrm{m}$, which is approximately $30 \%$ less than for the non-modified SP41 magnet.
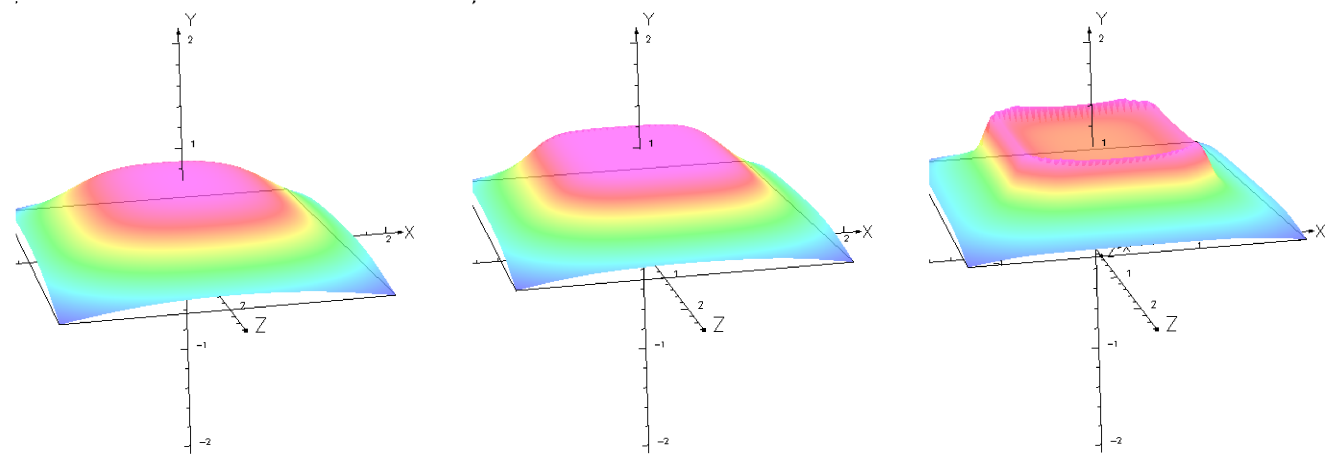

Figure 4: From left to right: $2 D$ distributions in $X Z$ plane of the magnetic field vertical component $B_{y}$ of modified SP41 magnet at $Y=0 \mathrm{~cm}$ (centre of the magnet), $Y=30 \mathrm{~cm}$, and $Y=50 \mathrm{~cm}$.

The $2 D$ distributions in $X Z$ plane of the magnetic field vertical component $B_{y}$ of the modified SP41 magnet at $Y=0 \mathrm{~cm}$ (centre of the magnet), $Y=30 \mathrm{~cm}$ and $Y=50 \mathrm{~cm}$ are presented in the left, middle, and right panels in Fig. 4. The field demonstrates very smooth behaviour except the region around pole of the magnet $(Y=50 \mathrm{~cm})$. The $3 D$ results for $B_{y}, B_{x}$ and $B_{z}$ components of the magnetic field were incorporated into the BM@N setup description for the simulation software [6].

\section{Commissioning of the SP41 magnet}

During 2012-2013 the warm SP41 dipole magnet has been significantly modernized. The magnet vertical gap has been enlarged by $30 \mathrm{~cm}$ up to $1.05 \mathrm{~m}$. The upper pole and upper horizontal beams have been filled by steel-15 in order to improve the magnetic field homogeneity. The upper coil (with reno- 
vated pole and horizontal beams) and lower coil alone have been rotated by $180^{\circ}$ in the horizontal plane to provide optimal access to the magnet infrastructure and detectors inside the BM@N experimental zone [1]. The magnet infrastructure, namely, pipes for cooling water, the current leads and diagnostics have been also rotated by $180^{\circ}$ and renovated. The view of modernized SP41 dipole magnet is presented in Fig.5.

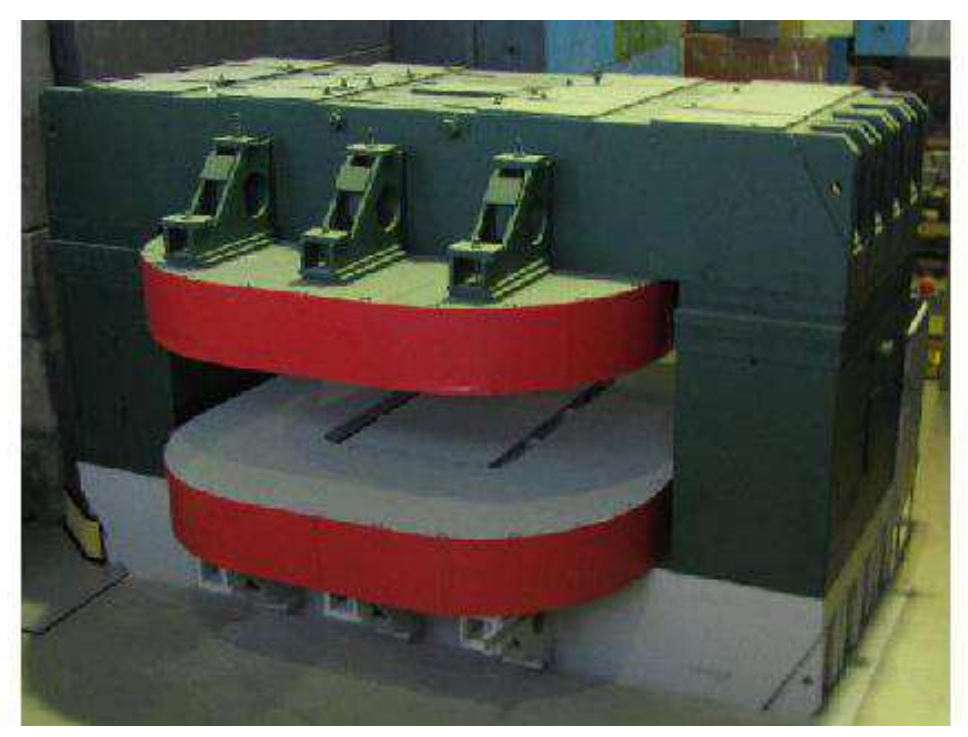

Figure 5: The view of modernized SP41 dipole magnet.

The commissioning of the SP41 magnet has been performed in two steps. Firstly, the operation of the magnet with the 1650 A current in the coils has been checked several times during several hours. Second step includes the measurements of the magnetic field in the centre of the magnet, the magnetic field integral and magnetic fringe field within working range of the current in the coils (up to $1960 \mathrm{~A}$ ).

The measurements of the $B_{y}$ vertical component of the magnetic field in the centre of modernized SP41 dipole magnet $(X=0, Y=0, Z=0)$ have been performed using planar Hall probe [10]. The $B_{y}$ in the centre of modernized SP41 dipole magnet as a function of the current in the coils is shown in Fig,6. The dashed curve is the result of the quadratic function approximation. The $B_{y}$ demonstrates the linear dependence on the current with negligible contribution of the quadratic term. The $B_{y}$ at $1900 \mathrm{~A}$ is equal to $0.933 \pm 0.003 \mathrm{~T}$ being in good agreement with the 3D TOSCA calculation shown in Fig, 2 , 


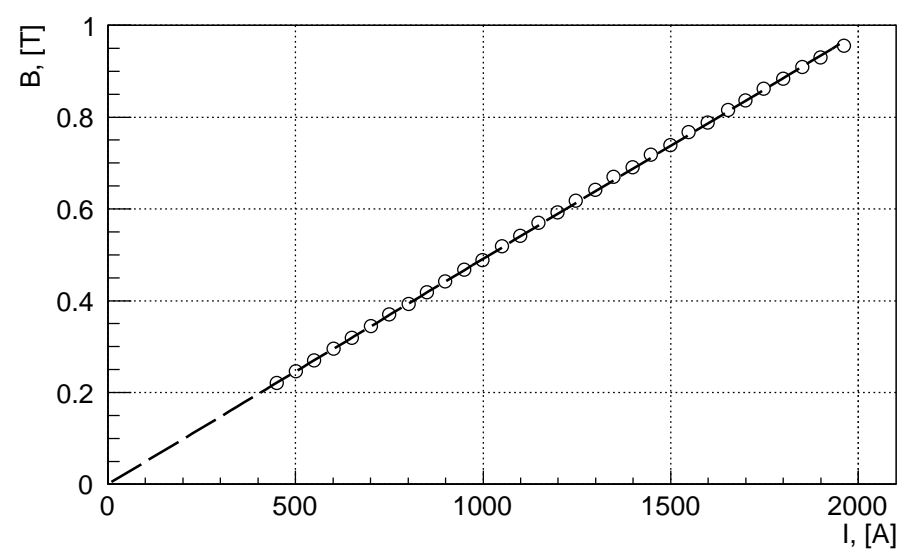

Figure 6: The $B_{y}$ vertical component of the magnetic field in the centre of modernized SP41 dipole magnet as a function of the current in the coils [10]. The dashed curve is the result of the quadratic function approximation.

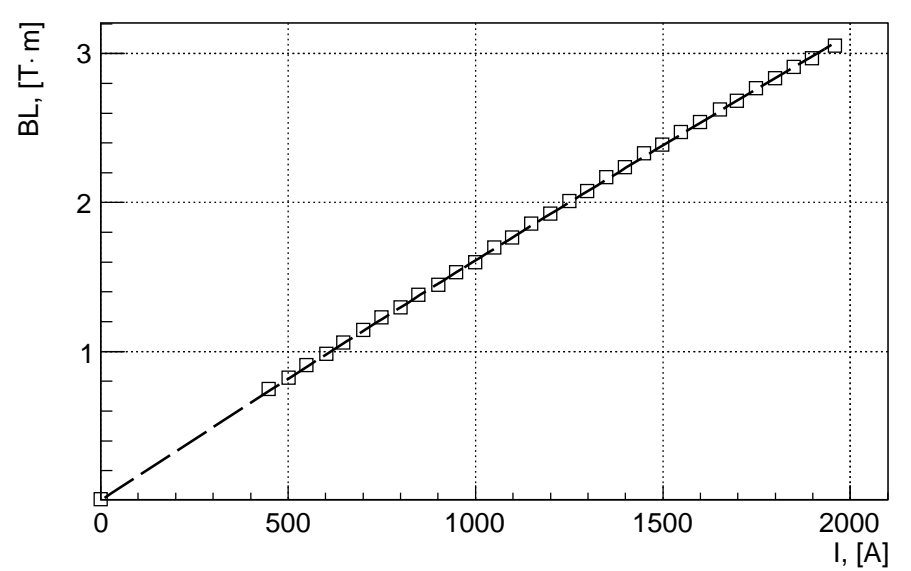

Figure 7: The magnetic field integral $\int B d l$ of modernized SP41 dipole magnet as a function of the current in the coils [10]. The dashed curve is the result of the quadratic function approximation. 
The measurements of the magnetic field integral $\int B d l$ of modernized SP41 dipole magnet has been performed using the method of the currentcarrying filament [10]. The magnetic field integral $\int B d l$ of modernized SP41 dipole magnet as a function of the current in the coils is shown in Fig.7. The dashed curve is the result of the quadratic function approximation. The magnetic field integral $\int B d l$ is equal to $2.986 \pm 0.009 \mathrm{~T} \cdot \mathrm{m}$ at $1900 \mathrm{~A}$. The nonlinear contribution is about $5 \%$. The measured value is in good accordance with the 3D TOSCA calculation results [1]. The field integral provides the bending angle of $\sim 70 \mathrm{mrad}$ for charged particles with $p / Z=13 \mathrm{GeV} / c$. On the other hand, since the saturation effect for the magnetic field integral is not observed at $1900 \mathrm{~A}$, the current value in the coils can be increased. This will provide larger bending angle and better momentum resolution.

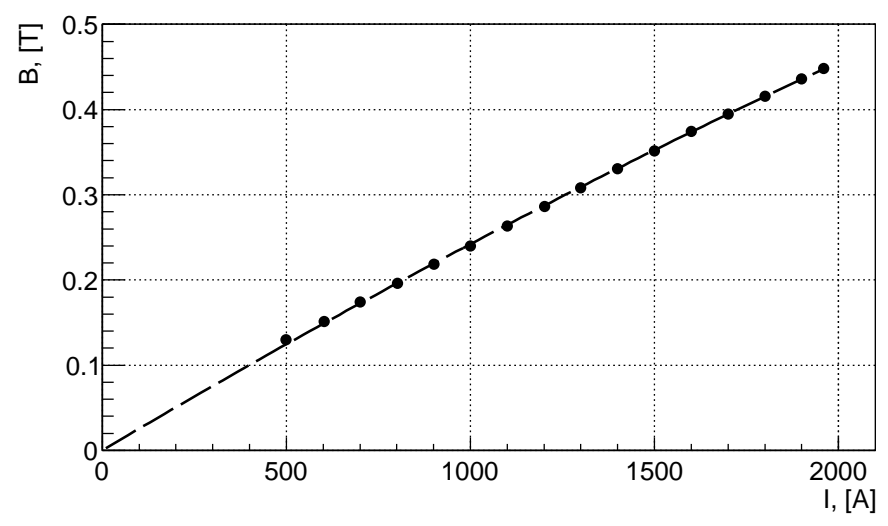

Figure 8: The magnetic field $|B|$ measured with the planar 3D Hall probe [1] at $X=0.2 \mathrm{~m}, Y=-0.22 \mathrm{~m}, Z=1.6 \mathrm{~m}$ as a function of the current in the coils. The dashed curve is the result of the quadratic function approximation.

The measurements of the magnetic field $|B|$ at $X=0.2 \mathrm{~m}, Y=-0.22 \mathrm{~m}$, $Z=1.6 \mathrm{~m}$ have been performed using planar 3D Hall probe described in details in ref.[1]. The position of this point is out of the magnet poles. The measured magnetic field $|B|$ as a function of the current value in the coils is shown in Fig.8. The dashed curve is the result of the quadratic function approximation. The $|B|$ demonstrates the non-negligible contribution of the quadratic term at large values of the current in the coils. For instance, the non-linear contribution is about $12 \%$ at the current value of $1900 \mathrm{~A}$. Therefore, the fringe field demonstrates the saturation effect at large currents in the coils, which is absent for the field in central region of the magnet. 


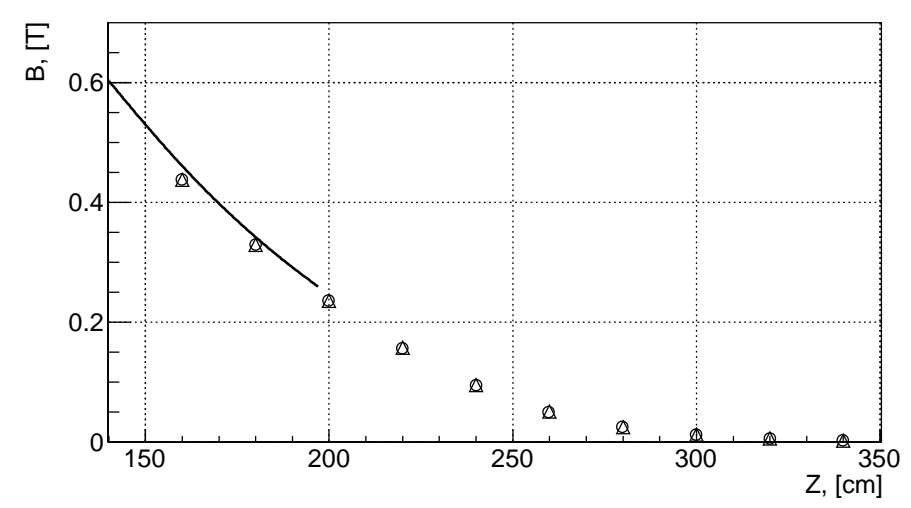

Figure 9: The magnetic field $|B|$ for the current value in the coils of $1900 \mathrm{~A}$ as a function of $Z$ coordinate $(X=0.2 \mathrm{~m}$ and $Y=-0.22 \mathrm{~m})$. The open circles and triangles are the data obtained with the planar and coaxial 3D Hall probes [1], respectively. The solid curve is the result of 3D TOSCA calculation scaled by factor 0.92 .

The measurements of the fringe magnetic field as a function of the distance from the magnet centre $Z$ at fixed values of $X=0.2 \mathrm{~m}$ and $Y=$ $-0.22 \mathrm{~m}$ coordinates have been performed using planar and coaxial 3D Hall probes [1]. The magnetic field $|B|$ for the current value in the coils of $1900 \mathrm{~A}$ as a function of $Z$ coordinate is shown in Fig 9, The open circles and triangles represent the data obtained with the planar and coaxial 3D Hall probes [1], respectively. One can see that the results obtained by these two probes are in good agreement. The solid curve is the result of 3D TOSCA calculation scaled by factor 0.92 . The shape of the measured magnetic field $|B|$ reproduces the TOSCA results. The observed difference can be recovered by the optimization of the SP41 dipole magnet model for the magnetic field TOSCA [9] calculations.

\section{Conclusions}

- The SP41 dipole magnet has been modernized for the studies of dense baryonic matter properties at Nuclotron. Namely, the magnet vertical gap has been enlarged up to $1.05 \mathrm{~m}$ to increase the angular acceptance for detection of hyperons [2, 5, 6], the magnetic field homogeneity improvement by the filling of the existed hole in the upper pole and 
horizontal beams by steel-15 has been achieved, the renovation of the magnet infrastructure has been made.

- The magnetic field in the centre of the magnet and out of the poles as well as field integral have been measured as a function of current value in the coils. The value of the magnetic field in the magnet centre and field integral at $1900 \mathrm{~A}$ are in good agreement with the results of 3D TOSCA calculation, while the measured fringe field at the same current is several percents below the calculated one.

- Further steps are the measurements of the magnetic field components along the optical axis of the SP41 magnet and 3D mapping of the magnetic field.

The authors thanks the technical services of LHEP participated in the modification of the SP41 dipole magnet, especially, G.S. Berezin, I.Ya. Nefediev, A.V. Shabunov, V.I. Sharapov. The authors are grateful to P.A. Rukoyatkin for the magnetic measurements, I.A. Bolshakova and S. Timoshin for their assistance in the use of 3D Hall probes. The work has been supported in part by RFBR under grant $N^{o} 13-02-00101$ a.

\section{References}

[1] Ablyazimov T. O. et al. (BM@N Collaboration) // Conceptual Design Report of BM@N. http://nica.jinr.ru/files/BM@N/BMN-CDR.pdf

[2] Ladygin V. P. et al. (BM@N Collaboration) // PoS Baldin-ISHEPPXXI. 2012. P.038.

[3] Bratkovskaya E. et al.// Nucl. Phys. A. 2013. V.914. P.387.

[4] Vasiliev T. A., Ladygin V. P. and Malakhov A. I. // Nucl. Phys. Proc. Suppl. B. 2011. V.219-220. P.312.

[5] Ladygin V. P., Jerusalimov A. P. and Ladygina N. B.// Phys. Part. Nucl. Lett. 2010. V.7. P.349.

[6] Ladygin V. P. et al.// In Proc. of the XV-th Advanced Research Workshop on High Energy Spin Physics (DSPIN-13), 8-12 October 2013, Dubna, Russia; Edited by A.V. Efremov and S.V. Goloskokov, JINR, Dubna, ISBN 978-5-9530-0315-3. 2014. P.239. 
[7] Terekhin A. A. et al.// PoS Baldin-ISHEPP-XXI. 2012. P.005.

[8] Piyadin S. M. et al. // Phys. Part. Nucl. Lett. 2012. V.9. P.589.

[9] OPERA-3d User Guide//http://www.lepp.cornell.edu/critten/opera/user-3d.pdf.

[10] Rukoyatkin P.A.// private communication. 